\title{
High-Efficiency Diffraction from Thin and Volume Dynamic Holograms in Linear Absorbers
}

\author{
I. N. Agishev and A. L. Tolstik \\ Belarussian State University, ul. Nezavisimosti 4, Minsk, 220030 Belarus \\ e-mail: tolstik@bsu.by
}

\begin{abstract}
The possibilities of improving the efficiency of light-field transformation by dynamic holograms using schemes of frequency-nondegenerate multiwave mixing in linearly absorbing liquids have been analyzed. The conditions are determined and the mechanism of the fifth-order nonlinearity related to the nonlinearity of the thermo-optical coefficient and the temperature dependence of the solvent density and specific heat is established.
\end{abstract}

DOI: $10.3103 / \mathrm{S} 1062873808120137$

\section{INTRODUCTION}

The expansion of the range of practical application of dynamic holograms in systems of real-time lightfield transformation and optical data processing is related to the optimization of holographic recording schemes and search for new methods aimed at attaining high diffraction efficiency. Along with the conventional use of media with cubic nonlinearity for holographic recording, the advantages of media with fifth- and higher order nonlinearity have been analyzed recently. Such media make it possible to implement multiwave mixing and different types of light-beam transformation (control of the wavefront shape, change in the propagation direction, image spatial filtration and frequency conversion, beam-to-beam data transfer, etc.) [1-3]. However, the application range of multiwave mixing is generally limited by low values of higher order nonlinearities.

In this study, we analyze the possibilities of increasing the efficiency of light-field transformation by dynamic holograms using the scheme of frequencynondegenerate multiwave mixing in linearly absorbing liquids, which were considered previously as having purely cubic nonlinearity. The conditions are determined and the mechanism of fifth-order nonlinearity related to the thermo-optical coefficient nonlinearity and temperature dependence of the density and specific heat of organic liquids is established.

\section{NONLINEAR HOLOGRAPHIC RECORDING IN LINEAR ABSORBERS}

The possibility of recording nonlinear dynamic holograms in pure organic liquids was noted for the first time in [4], where six-wave mixing in ethanol was implemented. To explain this effect, it was proposed to take into account the temperature dependence of the thermo-optical characteristics, which, according to [4], can by responsible for the saturating character of thermal nonlinearity, by analogy with the resonant media characterized by saturating absorption. However, such a phenomenological introduction of the saturation effect hinders quantitative determination of the nonlinearity parameter.

To describe the diffraction from thermal dynamic gratings at linear absorption, we propose to use the expansion of the functional temperature dependence of the refractive index in a Taylor series in derivatives of different orders and take into account the temperature dependence of the density and specific heat of the organic liquid used. In this case, the thermal change in the refractive index can be written as

$$
\Delta n=(\partial n / \partial T) \Delta T+\frac{1}{2}\left(\partial^{2} n / \partial T^{2}\right)_{\mathrm{eff}}(\Delta T)^{2}+\ldots
$$

where $\partial n / \partial T$ and $\partial^{2} n / \partial T^{2}$ are the first- and second-order thermo-optical coefficients, $\Delta T=k I \tau / C_{\rho}$ is a change in temperature, $k$ is the absorption coefficient, $I$ is the intensity, $\tau$ is the pulse width, and $C_{\rho}$ is the specific heat per unit volume. Expression (1) contains the effective second-order thermo-optical coefficient, which takes into account the temperature dependence of the specific heat per unit volume: $\left(\partial^{2} n / \partial T^{2}\right)_{\text {eff }}=\partial^{2} n / \partial T^{2}-$ $\left(\partial C_{\rho} / \partial T\right)(\partial n / \partial T) C_{\rho}^{-1}$.

The first term in (1) describes the cubic nonlinearity,

$$
\chi^{(3)}=\frac{c n_{0}^{2} k \tau}{16 \pi^{2} C_{\rho}}(\partial n / \partial T),
$$

which determines the efficiency of four-wave mixing, whereas the second term corresponds to the quadratic dependence of the refractive index on intensity and is 


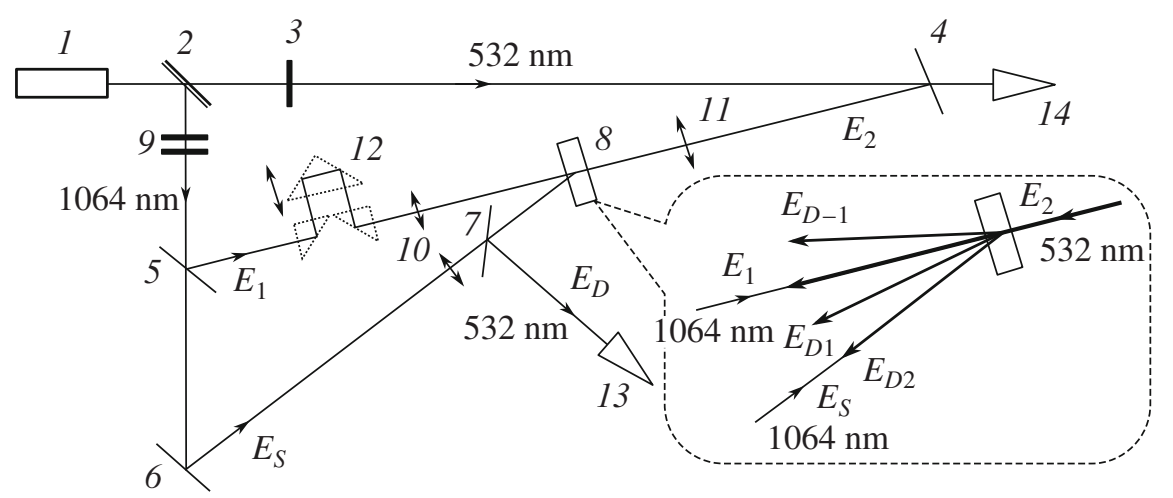

Fig. 1. Schematic of the experimental setup: (1) YAG laser, (2) beam splitter, $(3,9)$ light filters, (4-7) mirrors, (8) nonlinear medium, $(10,11)$ lenses, $(12)$ optical delay line, and $(13,14)$ recording system. The inset shows a schematic diagram of diffraction of the $E_{2}$ wave from a grating recorded by the waves $E_{1}$ and $E_{S}$.

unambiguously related to the fifth-order nonlinearity and six-wave mixing:

$$
\chi^{(5)}=\frac{c^{2} n_{0}^{3} k^{2} \tau^{2}}{256 \pi^{3} C_{\rho}^{2}}\left(\partial^{2} n / \partial T^{2}\right)_{\mathrm{eff}} .
$$

The obtained expressions (1)-(3) make it possible to calculate the diffraction efficiency of the recorded holographic grating. Depending on the recording conditions (relation between the layer thickness and lattice constant), holograms are classified as thin and volume in theory. When thin phase holograms are recorded, their diffraction efficiency is determined from the known formula [5]

$$
\xi=I_{D} / I_{2}=J_{M}^{2}(2 \pi L \Delta n / \lambda),
$$

where $J_{M}$ is the $M$-order Bessel function, corresponding to the $M$ th diffraction order, $L$ is the nonlinear layer length, and $\lambda$ is the radiation wavelength.

To analyze the efficiency of volume holograms, it is expedient to use the multiwave mixing formalism [6]. A diffracted wave $E_{D}$ is determined by the nonlinear polarization $P=\chi^{(N-1)}\left(E_{1} E_{S}^{*}\right)^{M} E_{2}(N$-wave mixing at $(N-1)$-order nonlinearity; $M=N / 2-1$ is the diffraction order); in this case, the propagation direction is determined by the phase-matching condition $\mathbf{k}_{D}=M \mathbf{k}_{1}-$ $M \mathbf{k}_{S}+\mathbf{k}_{2}$. Bragg diffraction into the $M$ th order occurs when the reading wave $E_{2}$ makes the angle $\beta=$ arc$\sin ((M / K) \sin (\alpha / 2))-\alpha / 2$ with the reference wave $E_{1}$, where $\alpha$ is the angle between the light beams recording a hologram; $K \omega$ is the reading wave frequency $(\beta \approx(M / K-1) \alpha / 2$ for small values of $\alpha)$. At $M=K$ (i.e., when the diffraction order coincides with the multiplicity order of the reading wave frequency), the matching condition is satisfied when the reading and reference waves are counterpropagating $[7,8]$.

To theoretically describe the multiwave mixing, we will use the approximation of slowly varying ampli- tudes, that gives a system of reduced wave equations for the interacting light fields [6]:

$$
\begin{gathered}
\frac{\partial E_{1, S}}{\partial r}=i \frac{2 \pi \omega}{c n_{0}}\left(E_{1, s} \chi_{0}(\omega)+E_{S, 1} \chi_{ \pm 1}(\omega)\right) \\
\frac{\partial E_{2, D}}{\partial r}=-i \frac{2 \pi K \omega}{c n_{0}}\left(E_{2, D} \chi_{0}(K \omega)+E_{D, 2} \chi_{\mp M}(K \omega)\right)
\end{gathered}
$$

where $\chi_{m}$ are the expansion components of the medium nonlinear susceptibility in the Fourier series expansion in dynamic-grating spatial harmonics: $\mathbf{K}=\mathbf{k}_{1}-\mathbf{k}_{S}$. Within the approximation of small diffraction efficiency, we can find from Eq. (6) that

$$
\frac{\partial E_{D}}{\partial r}=-i \frac{2 \pi K \omega}{c n_{0}} \chi^{(N-1)}\left(E_{1} E_{S}^{*}\right)^{M} E_{2},
$$

and derive an expression for the diffraction efficiency from (7):

$$
\xi=I_{D} / I_{2}=\left(\frac{2 \pi K \omega L}{c n_{0}}\right)^{2}\left|\chi^{(N-1)}\right|^{2}\left(I_{1} I_{S}\right)^{M}\left(\frac{8 \pi}{c n_{0}}\right)^{2 M} .
$$

\section{EXPERIMENTAL REALIZATION OF MULTIWAVE MIXING IN LINEAR ABSORBER}

Diffraction from dynamic gratings in linear absorbers was experimentally realized in the process of frequency-nondegenerate six-wave mixing; in this case a hologram is recorded at the fundamental frequency of a YAG laser $(\lambda=1064 \mathrm{~nm})$ and reading is performed at the second-harmonic frequency $(\lambda=532 \mathrm{~nm})$. The schematic of the experimental setup is shown in Fig. 1. Beam splitter 2 is used to separate the beams. Mirrors 5 and 6 and lens 10 form reference $\left(E_{1}\right)$ and signal $\left(E_{S}\right)$ waves recording a dynamic grating in a nonlinear medium 8 at the beam diameter $d=650 \mu \mathrm{m}$. The wave intensity is changed by light filters 9 . To synchronize the arrival time of the recording laser pulses, we used a 

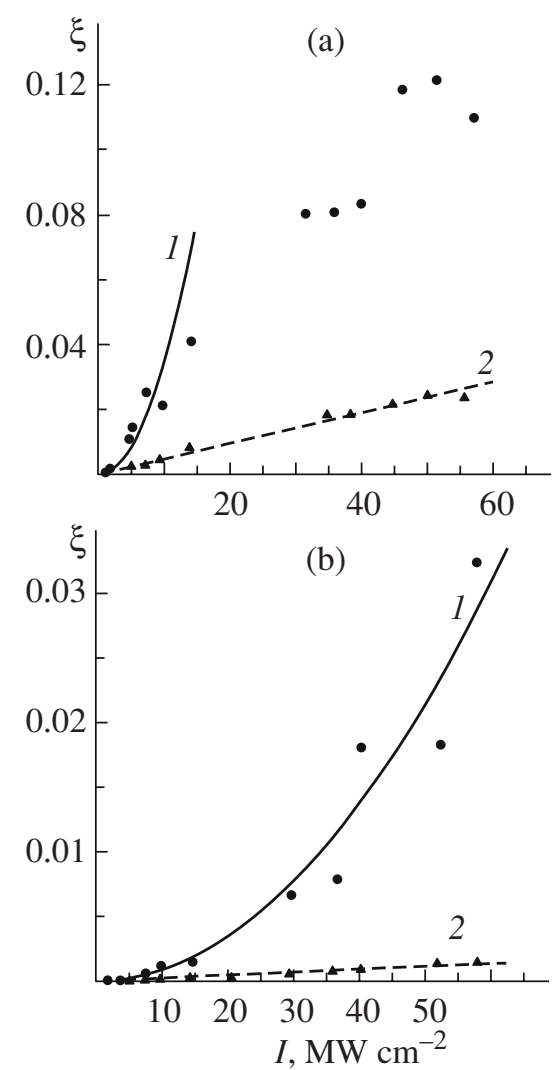

Fig. 2. Dependence of the diffraction efficiency $\xi$ on the intensity $I=I_{1}=I_{S}$ of the waves recording a hologram for the diffraction orders (1) +1 and (2) -1 from gratings with periods $\Lambda=$ (a) 20 and (b) $6 \mu \mathrm{m}$ at the optical thickness $k L=$ 1 of the medium.

delay line 12 . The reading wave $E_{2}$ was directed counter to the recording waves by mirror 4 , and lens 11 formed a beam of the same diameter $(d)$ in the medium. To measure the diffraction efficiency of the dynamic hologram, we used a detection system based on photodiodes 13 and 14. An ethanol solution of copper chloride (with high thermal nonlinearity) was chosen as a linearly absorbing medium. Its advantage is a high absorption in the near-IR range as compared to the visible range (the absorption coefficients at wavelengths of 1064 and $532 \mathrm{~nm}$ differ by more than an order of magnitude).

We analyzed experimentally the diffraction from transmission gratings of different periods $\Lambda$ (from 6 to $20 \mu \mathrm{m})$; such a change in the period corresponded to a decrease in the Klein parameter $Q=2 \pi \lambda d / n \Lambda^{2}$ by an order of magnitude (from 40 to 4 ). The value $Q=40$ corresponds to the volume grating criterion, while $Q=4$ is an intermediate case, where properties of both planar and volume hologram recording should manifest themselves. However, several diffraction orders (schematically shown in Fig. 1) with rather different diffracted-wave intensities were observed in all cases.
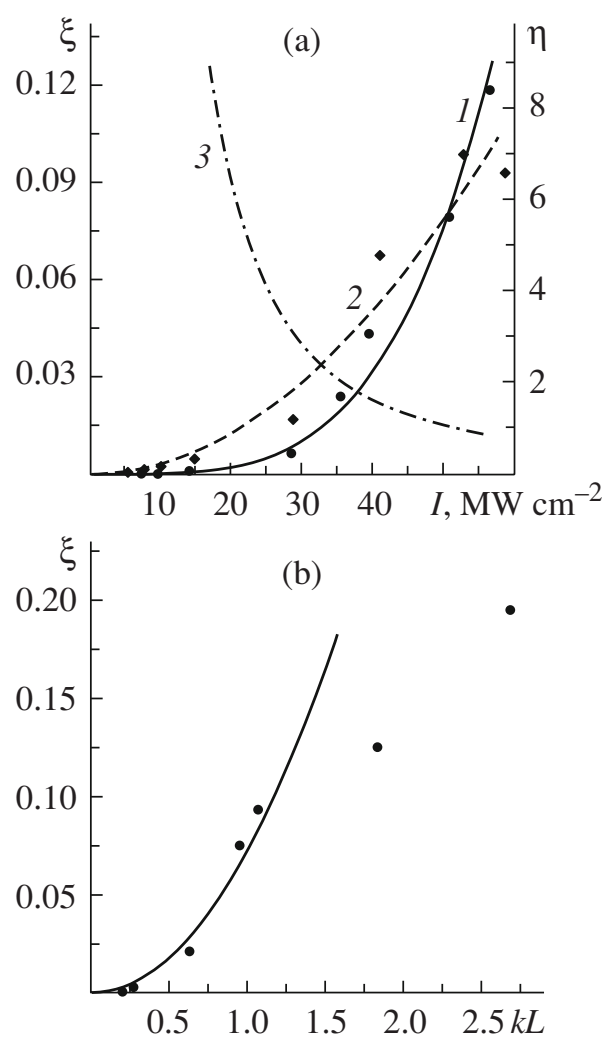

Fig. 3. Dependence of the second-order diffraction efficiency $\xi$ on (a) the intensity $I=I_{1}=I_{S}$ of the waves recording a hologram at $k L=1$ and (b) the optical thickness $k L$ of the medium at $I=50 \mathrm{MW} \mathrm{cm}$ for the gratings with the periods $\Lambda=$ (1) 6 and (2) $20 \mu \mathrm{m}$. The dot-dashed line (3) shows the ratio of the grating diffraction efficiencies: $\eta=\xi(\Lambda=$ $20 \mu \mathrm{m}) / \xi(\Lambda=6 \mu \mathrm{m})$.

Figures 2 and 3 show the dependences of the diffraction efficiency $\xi$ on the intensity $I=I_{1}=I_{S}$ of the waves recording a hologram and on the optical thickness $k L$ of the medium, which were obtained at the 1- and 2-order diffraction from gratings with different periods. As one would expect, with an increase in the Klein parameter $Q$ on going from a grating with the period $\Lambda=20 \mu \mathrm{m}$ (Fig. 2a) to that with $\Lambda=6 \mu \mathrm{m}$ (Fig. 2b) the \pm 1 -order diffraction efficiency is decreased. At the same time, the differences for the orders +1 and -1 increase; the reason is that the order +1 is closer to the propagation direction of the diffracted wave $E_{D 2}$, corresponding to the Bragg condition. A more interesting fact is the existence of a range of intensities where the secondorder diffraction is stronger for a grating with a smaller

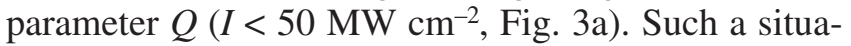
tion can be explained by different asymptotics of the dependences of diffraction efficiency on intensity for both gratings. The diffraction efficiency for a grating with a large period $(\Lambda=20 \mu \mathrm{m}, Q=4)$ is described well by a quadratic function (Fig. 3a, dashed line). For a grating with $\Lambda=6 \mu \mathrm{m}(Q=40)$, a good approximation is a fourth-order parabola (solid line). 
Note that, for small values of the diffraction efficiency $\xi$, its dependence on the optical thickness $k L$ of the medium, according to Eq. (8), is described by a quadratic parabola (Fig. 3b). The increase in the diffraction efficiency tends to a constant value at the optical thickness $k L=1$, which corresponds to $\xi \approx 10 \%$ for a volume grating. The maximum diffraction efficiency was $20 \%$, that exceeds by several orders of magnitude the values obtained in the previous experimental studies on the frequency-nondegenerate six-wave mixing using twophoton absorption in polymers [9], saturating absorption in solutions of organic dyes [6], and thermal nonlinearity of pure organic liquids [4]. For comparison, we should note that, using the four-wave mixing scheme, we realized recording of transmission phase holograms with a maximum diffraction efficiency of $60 \%$ in an ethanol solution of copper chloride.

Note also that the adequacy of the proposed approach to description of dynamic holograms in linear absorbers is confirmed by comparison of the obtained experimental data with the results of theoretical calculations. In particular, using the measured diffraction efficiency and Eqs. (3) and (8), we estimated the effective second-order thermo-optical coefficient to be $\left(\partial^{2} n / \partial T^{2}\right)_{\text {eff }} \approx 10^{-6} \mathrm{~K}^{-2}$. At the same time, the tabular data [10] give the ethanol specific heat per unit volume $C_{\rho}=$ $1.92 \mathrm{~J} /\left(\mathrm{K} \mathrm{cm}^{3}\right)$, its derivative $\partial C_{\rho} / \partial T \approx 5 \times 10^{-3} \mathrm{~J} /\left(\mathrm{K}^{2} \mathrm{~cm}^{3}\right)$, and the thermo-optical coefficient $\partial n / \partial T=-4 \times 10^{-4} \mathrm{~K}^{-1}$. The estimation using the Lorentz-Lorenz formula for the second-order thermo-optical coefficient is $\partial^{2} n / \partial T^{2} \approx$ $-10^{-7} \mathrm{~K}^{-2}$. Thus, the known data give the following estimate of the effective thermo-optical coefficient: $\left(\partial^{2} n / \partial T^{2}\right)_{\text {eff }}=\partial^{2} n / \partial T^{2}-\left(\partial C_{\rho} / \partial T\right)(\partial n / \partial T) C_{\rho}^{-1} ;$ it is similar to the experimentally obtained value.

\section{CONCLUSIONS}

Good prospects of linearly absorbing colored organic liquids for high-efficiency six-wave mixing at the fifth-order nonlinearity have been demonstrated. It is established that the thermal nonlinearity caused by the temperature dependence of the solvent density and specific heat makes a decisive contribution to mixing. A concept of the effective second-order thermo-optical coefficient was introduced to take into account the above-mentioned dependences making it possible to develop an adequate theoretical model.

\section{REFERENCES}

1. Merkel, K.D., Cole, Z., and Babbitt, W.R., J. Lumin., 2000, vol. 86, p. 375.

2. Astinov, V., Kubarych, K.J., Milne, C.J., and Miller, R.J.D., Opt. Lett., 2000, vol. 25, no. 11, p. 853.

3. Ormachea, O., Romanov, O.G., Tolstik, A.L., et.al., Opt. Express., 2006, vol. 14, no. 18, p. 8298.

4. Ormachea, O. and Tolstik, A.L., Izv. Ross. Akad. Nauk, Ser. Fiz., 2007, vol. 71, no. 1, p. 131.

5. Miller, M., Golografiy (Holography), Leningrad: Mashinostroenie, 1979.

6. Rubanov, A.S., Tolstik, A.L., Karpuk, S.M., and Ormachea, O., Opt. Commun., 2000, vol. 181, nos. 1-3, p. 183.

7. Ivakhnik, V.V. and Nikonov, V.I., Opt. Spectrosk., 1993, vol. 75 , no. 2 , p. 385 .

8. Karpuk, S.M., Rubanov, A.S., Tolstik, A.L., and Chalei, A.V., Pis'ma Zh. Tekh. Fiz., 1994, vol. 20, no. 12, p. 4.

9. Charra, F. and Nunzi, J.-M., J. Opt. Soc. Amer. B, 1991, vol. 8, no. 3, p. 570 .

10. Stabnikov, V.I., Roiter, I.M., and Protsyuk, T.B., Etilovyi spirt (Ethanol), Moscow: Pishchevaya Promyshlennost', 1976. 